\title{
Enquête De Satisfaction Des Usagers AMO A L'hôpital Gabriel TOURE Et Au Centre De Santé De Référence De La Commune 1 En 2018
}

\author{
Guindo Oumar ${ }^{*}$, Diallo Hawa Mamadou ${ }^{2}$, Niambele Maimouna ${ }^{2}$, Coulibaly Abdouramane ${ }^{3}$, Sogoba Mahamadou ${ }^{4}$,
} Berthe Mamadou ${ }^{5}$, Diop Samba ${ }^{3}$

${ }^{1}$ Hôpital Sominé DOLO de Mopti, Sévaré, Mali

${ }^{2}$ Faculté de Médecine et d'Odontostomatologie, Bamako, Mali

${ }^{3}$ Département d'enseignement et de recherche en santé publique, Bamako, Mali

${ }^{4}$ Ministère de la santé et des affaires sociales, Bamako, Mali

${ }^{5}$ Direction générale de la santé et de l'hygiène publique, Bamako, Mali

DOI: $10.36347 /$ SJAMS.2020.v08i05.004

| Received: 19.11.2019 | Accepted: 26.11.2019 | Published: 06.05.2020

*Corresponding author: Oumar Guindo

\section{Abstract}

This work involves an evaluation of compulsory health insurance in Mali at the level of two health establishments in Bamako in 2018 in order to assess user satisfaction with the implementation and functionality of the health insurance scheme. To do this, a questionnaire was applied to 200 AMO users who requested one of the delivery services in the targeted institutions. This questionnaire collected their perceptions on the reception, orientation in the institutions, waiting time, listening and communication with service providers, and the quality of services. Overall, it emerged that $60 \%$ of the users surveyed are satisfied with the implementation and functionality of health insurance in the target institutions. Thus 153/200 users found the reception satisfactory, 167/200 were satisfied with the orientation in the facilities, 185/200 and 160/200 users respectively were satisfied with the communication with the staff and their listening, 151/200 users were nevertheless not satisfied with the waiting time. In the end, 189/200 users satisfactorily assessed the quality of care in the two facilities.

Keywords: users, satisfaction, AMO.

Copyright @ 2020: This is an open-access article distributed under the terms of the Creative Commons Attribution license which permits unrestricted use, distribution, and reproduction in any medium for non-commercial use (NonCommercial, or CC-BY-NC) provided the original author and source are credited

\section{INTRODUCTION}

L'accessibilité permanente, géographique et financière des populations à des soins de santé de qualité demeure un objectif prioritaire pour la politique sectorielle de santé et de population du Mali depuis l'adoption de ladite politique en 1990.

Cette politique a été matérialisée par l'élaboration d'un premier plan décennal d'où ont été issus deux plans quinquennaux dont la mise en œuvre a abouti à la construction et / ou la revitalisation des établissements de santé existants, au renforcement des équipements et à la dotation desdits établissements en personnel qualifié[1].

Dans la même lancée, des documents de normes, de procédures et de politiques ont été élaborés et diffusés pour mieux coordonner et orienter les interventions et les pratiques professionnelles [1].
En dépit de ces importants efforts du Gouvernement et des partenaires, la demande des services de santé, de façon générale, est restée faible [1]. En effet, selon l'annuaire du système national d'information sanitaire 2015, le taux d'utilisation des services était de 0,41 nouveau contact/habitant/an, le taux de recours en CPN 3 des femmes enceintes était de $39 \%$ et la prévalence contraceptive de $8,88 \%$ ) [2].

Les raisons de la faible demande de santé tiennent à plusieurs facteurs dont ceux socioculturels, le sous financement des services de santé et le faible pouvoir d'achat de la population [3-6].

Au Mali, comme dans la plupart des pays africains, les ménages sont les principales sources de financement du système de santé à travers des mécanismes de paiement direct responsables de leur appauvrissement [7-9]. 
Les chiffres des comptes nationaux de la santé (CNS) montrent que les paiements directs des ménages pour les services prioritaires sont nettement plus importants que ne le pensent les gouvernements ou les donateurs. Le coût des soins de santé, notamment les paiements directs des soins, constitue ainsi donc un obstacle majeur à l'accès aux services de santé $[3,5]$.

Le sous-financement de la santé et le poids élevé des dépenses de santé pour les ménages ont amené le Gouvernement du Mali à s'engager sur de nouvelles perspectives dont l'avènement de l'assurance maladie obligatoire (AMO) le 26 juin 2009 [3, 5, $12,13]$.

Le présenta article se propose d'apprécier la satisfaction des usagers AMO de deux établissements de santé de Bamako sur la mise en œuvre et la fonctionnalité de l'AMO en 2018.

\section{Materiels et Methode}

Pour réaliser ce travail, 200 usagers de l'AMO en provenance des deux établissements ont été interviewé sur la qualité de l'accueil, le temps d'attente, la qualité des soins, les insuffisances perçues et les propositions d'amélioration.

Un questionnaire a été appliqué aux usagers après une sélection aléatoire en les interrogeant soit dans la cour soit à la sortie de l'établissement afin de recueillir leurs impressions à travers un entretien semi directif.

\section{RESULTATS}

L'interview des usagers AMO au niveau de l'hôpital Gabriel et du centre de santé de référence de la commune1 a abouti au constat de leur satisfaction globale par rapport à la mise en œuvre et à la fonctionnalité de l'AMO.

Ainsi la majorité des usagers enquêtés 78,5\% ont été satisfaits de la qualité de l'accueil dans les structures, $80 \%$ de la bonne écoute du personnel et 92,5 $\%$ de la communication sur la maladie ou le traitement. Par ailleurs une majorité des usagers $75,5 \%$ ne sont pas satisfaits du temps d'attente dont $38 \%$ trouvent que ce temps d'attente est prolongé et $17 \%$ très prolongé. La qualité des soins quant à elle a été appréciée avec satisfaction par $94,5 \%$ des usagers.

Table-1: Récapitulatif des éléments de satisfaction des usagers de l'hôpital Gabriel TOURE par rapport à la mise en œuvre de l'AMO

\begin{tabular}{|c|c|c|c|c|c|c|}
\hline Satisfaction des usagers & $\mathbf{0}$ & 1 & 2 & 3 & 4 & 5 \\
\hline $\begin{array}{l}\text { Perception de la qualité de l'accueil par l'usager dès son entrée dans la structure et } \\
\text { jusqu'à sa sortie }\end{array}$ & & & & $\mathrm{X}$ & & \\
\hline $\begin{array}{l}\text { Présence de panneaux ou plaques signalétiques ou d'affiches permettant de guider } \\
\text { dans la cour de l'établissement hospitalier }\end{array}$ & & & & & $\mathrm{X}$ & \\
\hline Disponibilité du prestataire à écouter attentivement l'usager & & & & $\mathrm{X}$ & & \\
\hline Respect de l'ordre d'arrivée et de consultation pour chaque usager & & & & & $\mathrm{X}$ & \\
\hline Temps d'attente avant le service est acceptable par l'usager & & $\mathrm{X}$ & & & & \\
\hline Les services sont offerts dans un cadre qui respecte l'intimité de l'usager & & & & & $\mathrm{X}$ & \\
\hline Bonnes pratiques des différents services avec professionnalisme & & & & & $\mathrm{X}$ & \\
\hline Etat d'hygiène de la cour, des services sollicités, pratique de soins avec hygiène & & $\bar{X}$ & & & & \\
\hline Niveau d'équipement du service permettant l'exécution correcte des taches & & & & $\mathrm{X}$ & & \\
\hline $\begin{array}{l}\text { Disponibilité des prestataires qualifiés à tout moment, disponibilité et } \\
\text { fonctionnalité des équipements }\end{array}$ & & & & $\mathrm{X}$ & & \\
\hline $\begin{array}{l}\text { La moyenne de la satisfaction des usagers par rapport à la mise en ouvre de } \\
\text { l'AMO }\end{array}$ & & & & $\mathbf{X}$ & & \\
\hline
\end{tabular}

Inférieur à $3=$ peu satisfaisant, 3 = satisfaisant et de 4 à $5=$ très satisfaisant 
Table-2: Récapitulatif des éléments de satisfaction des usagers du centre de santé de référence de la commune 1 par rapport à la mise en œuvre de l'AMO

\begin{tabular}{|c|c|c|c|c|c|c|}
\hline Satisfaction des usagers & $\mathbf{0}$ & 1 & 2 & 3 & 4 & 5 \\
\hline $\begin{array}{l}\text { Perception de la qualité de l'accueil par l'usager dès son entrée dans la structure et } \\
\text { jusqu'à sa sortie }\end{array}$ & & & & & $\mathrm{X}$ & \\
\hline $\begin{array}{l}\text { Présence de panneaux ou plaques signalétiques ou d'affiches permettant de guider dans la } \\
\text { cour de l'établissement hospitalier }\end{array}$ & & & & & $\mathrm{X}$ & \\
\hline Disponibilité du prestataire à écouter attentivement l'usager & & & & & $\mathrm{X}$ & \\
\hline Respect de l'ordre d'arrivée et de consultation pour chaque usager & & & & & $\mathrm{X}$ & \\
\hline Temps d'attente avant le service est acceptable par l'usager & & & & $\mathrm{X}$ & & \\
\hline Les services sont offerts dans un cadre qui respecte l'intimité de l'usager & & & & & $\mathrm{X}$ & \\
\hline Bonnes pratiques des différents services avec professionnalisme & & & & & $\mathrm{X}$ & \\
\hline Etat d'hygiène de la cour, des services sollicités, pratique de soins avec hygiène & & & $\mathrm{X}$ & & & \\
\hline Niveau d'équipement du service permettant l'exécution correcte des taches & & & & $\mathrm{X}$ & & \\
\hline $\begin{array}{l}\text { Disponibilité des prestataires qualifiés à tout moment, disponibilité et fonctionnalité des } \\
\text { équipements }\end{array}$ & & & & $\mathrm{X}$ & & \\
\hline La moyenne de la satisfaction des usagers par rapport à la mise en œuvre de l'AMO & & & & $\mathbf{X}$ & & \\
\hline
\end{tabular}
$1=20 \% \quad 2=40 \% \quad 3=60 \% \quad 4=80 \% \quad 5=100 \%$

Inférieur à $3=$ peu satisfaisant, $3=$ satisfaisant et de 4 à $5=$ très satisfaisant

\section{Commentaires et Discussions}

Dans notre étude nous nous sommes intéressés à plusieurs entités qui rentrent dans le cadre de la satisfaction des usagers. Les usagers de façon globale, qu'il s'agisse des assurés ou non, sont au centre de toute activité de soins de santé, en ce sens que tout ce qui est entrepris dans le cadre du développement socio sanitaire est fait dans l'intérêt des usagers [14].

Le recueil de l'opinion des usagers sur l'efficacité d'un service est déterminant quant à l'avenir et à l'utilisation de ce service [14].

Concernant spécifiquement l'accueil, selon Y Jaffrey, l'accueil s'entend au sens strict, c'est-à-dire en référence aux manières de recevoir les gens, de les écouter et de leur parler en tant qu'individu, ainsi pour les usagers des services publics de santé, les indicateurs de la qualité et de l'équité dans l'accès aux soins se mesurent à l'accueil et aux soins dispensés [15]. Notre étude a abouti à un constat de satisfait des usagers par rapport à l'accueil avec un taux de $60 \%$.

L'étude de Daou a démontré que les patients restent toujours sensibles à la qualité de l'accueil au respect de l'ordre d'arrivée et au temps d'attente dans les structures de soins [15].

Au cours de leur interview certains usagers ont énumérés quelques insuffisances à la mise en œuvre de l'assurance maladie, il 's'agit de:

- l'insuffisance du nombre de guichet AMO par rapport à l'affluence des usagers,

- l'absence de certains médicaments prescrit par les prestataires sur la liste de médicament prise en charge par l'AMO et le paiement d'une différence de prix sur certains médicaments ;

- le nombreux aller et retour des usagers entre les services et le guichet pour la validation des feuilles de soin entrainant un temps d'attente prolongé;
- la mauvaise qualité de l'accueil de la part de certains personnels des guichets;

- la limitation de l'âge des ayants droits à 21 ans;

- la fermeture des droits des usagers sans leur information préalable.

\section{CONCLUSION}

A la lumière de nos résultats, il est ressorti globalement que les usagers AMO sont satisfaits de la mise en œuvre et de la fonctionnalité de l'AMO à l'hôpital Gabriel TOURE et au centre de santé de référence de la commune 1 .

Ainsi, par rapport à l'accueil dans les établissements, il ressort que plus de la majorité des usagers ont été satisfaits de la qualité de l'accueil dans les deux structures.

Concernant l'information sur la maladie et le traitement, les usagers enquêtés ont reçu dans la majorité des cas auprès des prestataires les informations sur leur maladie et le traitement. Ainsi la qualité des soins telle que perçue par les mêmes usagers est satisfaisante dans la majorité des cas.

Par rapport au temps d'attente une majeure partie des usagers enquêtés ne sont pas satisfaits de ce temps d'attente qui est prolongé pour beaucoup d'entre eux.

De l'avis de certains usagers, il existe quelques insuffisances relatives à la fermeture de leur droit sans information préalable, au paiement d'une différentielle de prix lors du paiement de certains médicaments prescrits. 
1. Cellule De Planification Et De Statistique Secteur Sante, Developpement Social Et Promotion De La Famille, Secretariat Permanent Du Prodess, Plan Décennal De Développement Sanitaire Et Social (PDDSS). 2014-2023: 13, 24, 25, 59.

2. Cellule De Planification Et De Statistique Secteur Sante, Developpement Social Et Promotion De La Famille. Annuaire 2015 Du Système National D'information Sanitaire Et Social. 2015: 11, 18, 22.

3. Ministère de la Santé, Institut National de Recherche en Santé Publique, Organisation mondiale de la Santé, Les Comptes Nationaux de la Santé du Mali 1999-2004, version finale 7 mars 2007, 96.

4. Banque Mondiale, La Problématique de la Santé et de la Pauvreté au Mali, Rapport Principal, 02 Avril. 2011(1)148:24, 77 à 96

5. Cellule De Planification Et De Statistique Secteur Santé, Développement Social Et Promotionde La Famille, Comptes de la santé du Mali, édition 2014, CPS/SSDSPF Mars. 2016: 22, 27.

6. Cellule De Planification et de Statistique (CPS/SSDSPF), Institut National de la Statistique (INSTAT/MPATP), INFO-STAT et ICF International, 2014. Enquête Démographique et de Santé au Mali 2012-2013. Rockville, Maryland, USA : CPS, INSTAT, INFO-STAT et ICF International, présentation Mali.2014; 31-35, 5159, 361-380

7. Banque Mondiale, Région Afrique, Département du Développement humain, La problématique de la santé et de la pauvreté au Mali, Analyse des indicateurs de santé et population dans le cadre de la stratégie nationale de lutte contre la pauvreté au Mali, Septembre. 2004, 246.
8. Laporte JD. Le financement communautaire des services de santé, la situation au Mali et dans d'autres Etats francophones d'Afrique, iuéd, octobre 2004, Institut universitaire d'études du développement, Service des publicationsCase postale $136 \quad-\quad \mathrm{CH}-1211$ GENÈVE 21www.iued.unige.ch publications@iued.unige.ch

9. Gilles Dussault, Pierre Fournier, Alain Letourmy. L'Assurance maladie en Afrique francophone, Améliorer l'accès aux soins et lutter contre la pauvreté, Editeurs. 2006: 41-42

10. Organisation Mondiale de la Santé. Rapport sur la santé dans le monde. 2008: 14 Octobre 2008, Almaty, Kazakhstan, 17.

11. Christopher Dye, Ties Boerma, David Evans. Rapport sur la santé dans le monde 2013 : la recherche pour la couverture sanitaire universelle. 9789240691193 (PDF), Organisation mondiale de la Santé 2013, Engagement. 2005: 22-25

12. Ministère de la Santé, Direction des Ressources Humaines, Profil des Ressources Humaines en Santé du Mali. 2009: 102.

13. Berthe YA, Et Balique H. Etude sur les ressources humaines du secteur de la santé au Mali, Ministère de la Santé de la République du Mali et Ministère des Affaires Etrangères de la République Française.

14. Ebrahim K, Abdelouahid EM, Abdelouahid I. Etude de satisfaction des « clients » d'un organisme gestionnaire d'assurance maladie au Maroc, Assurances et gestion des risques. 2007; 75(3).

15. Daou B, Analyse de l'offre de soins dans le cadre de la mise en œuvre de l'assurance maladie obligatoire (AMO) au Mali, mémoire CESAG Dakar. 2011: 68 . 